\title{
The Development of the Internalization of Sociocultural Body Ideals Scale Using Items From the Sociocultural Attitudes Towards Appearance Questionnaire: Validity, Reliability, and Measurement Invariance Testing
}

\author{
Alvaro Sicilia $^{1,2}$ - Antonio Granero-Gallegos ${ }^{1,2}$ - Manuel Alcaraz-lbáñez ${ }^{1,2}$. \\ Mark D. Griffiths ${ }^{3}$ (D) - Isabel Sánchez-Gallardo ${ }^{4}$. Jesús Medina-Casaubón ${ }^{4}$
}

Published online: 22 October 2019

(C) The Author(s) 2019

\begin{abstract}
Eating disorders and body image disturbance are known to result in unhealthy consequences. When attempting to understand the etiology and management of these disorders, sociocultural models of body image disturbance and disordered eating have been predominant. The objective of the present study was to develop and validate the Internalization of Sociocultural Body Ideals Scale (ISBIS), a psychometric instrument that utilizes items from various versions of the Sociocultural Attitudes Towards Appearance Questionnaire (SATAQ). The ISBIS was developed to overcome some of the limitations found in existing instruments (including the SATAQ) that assess the internalization of sociocultural body ideals. The sample comprised 1130 students aged between 10 and 14 years. The psychometric properties of the ISBIS were examined using different analyses. The results supported the eight-item structure: four items for thin body ideal internalization and four items for thin body muscular/athletic internalization. The structure showed invariance to gender and achieved acceptable internal consistency and temporal stability indexes. Controlling for gender and body mass index, the results also showed that the internalization of the thin body ideal positively predicted social-physique anxiety in a statistically significant way. The results provide empirical evidence that the ISBIS is a reliable and valid instrument for assessing sociocultural body ideals in Spanish preadolescents.
\end{abstract}

Keywords Thin body ideal $\cdot$ Muscular/athletic body ideal $\cdot$ Social physique anxiety $\cdot$ Body image Preadolescence

Research has consistently associated eating disorders and body image disturbance with unhealthy consequences such as depressive symptoms and functional impairment (Stice et al. 2011; Thompson and Stice 2001). A challenge for researchers is to determine the risk

Mark D. Griffiths

mark.griffiths@ntu.ac.uk

Extended author information available on the last page of the article 
factors underlying these disorders and to intervene and reduce their effects to promote a healthy lifestyle (Stice et al. 2011). Sociocultural models of body image disturbance and disordered eating have been predominant when attempting to understand the etiology and the management of these disorders and have received strong empirical support from crosssectional longitudinal and meta-analytic studies (Bassett-Gunter et al. 2017; Stice et al. 1998). Sociocultural models posit that individuals feel pressure from diverse sources (e.g. family peers media and partners) to attain an ideal body defined by the sociocultural patterns of beauty belonging to western countries (McCabe et al. 2015).

Traditionally, the literature that has examined social pressure has centered upon women and the consequences of searching for ideal of feminine beauty associated with thin-bodied models (Cusumano and Thompson 1997; Thompson and Stice 2001; Thompson et al. 2004). This may be explained by the fact that women are more likely to be the targeted by their peers and the media to have "perfect bodies" compared with men leading to more negative consequences (e.g., eating disorders) among women than in men (Dakanalis et al. 2017). Despite this assumption, evidence demonstrates that men also feel sociocultural pressure to achieve socially accepted standards of beauty (Karazsia and Crowther 2008; Ricciardelli and McCabe 2004; Tylka 2011). Indeed, recent research has examined the variables related to sociocultural pressure felt by males in search of an ideal body associated with the athletic/ muscular-bodied model, which is related to the culture of masculinity and the role of the manly figure (Edwards et al. 2016; Thompson et al. 2012).

Theoretically, social pressure towards these body ideals carries negative consequences for the individual to the extent that they internalize and accept such attitudes as their own, attitudes that are approved by significant others (e.g., parents, peer group, teachers, health service providers). Here, the internalization process refers to the extent that individuals cognitively "buy into" the culturally sanctioned ideals of beauty (Thompson and Stice 2001), so that they end up assuming such ideals as their own standard. The internalization of beauty ideals (which are generally unattainable for the individual) has been shown to be a risk factor for unhealthy behaviors associated with body image disturbance and eating disorders (Dye 2016). Furthermore, it is important to highlight that although action to change the pressure of the different social agents of body image are necessary, research indicates it might be more feasible to intervene in the internalization process of socially sanctioned ideals as a risk factor associated with body dissatisfaction (Dittmar and Howard 2004). Given the importance of body ideal internalization for the prevention and treatment of eating disorders and other unhealthy behaviors, it is essential to have appropriate and sensitive psychometric measures to assess this construct.

To date, there have been two scales developed that assess the internalization of body ideals - the Ideal Body Internalization Scale (Stice et al. 1994) and the Sociocultural Attitudes Towards Appearance Questionnaire (SATAQ; Heinberg et al. 1995). The most recent revised version of the IBIS (IBIS-R; Stice 2001; Stice et al. 1996) is a unidimensional measure that was developed using six items from the Ideal Body Stereotype Scale (Stice et al. 1994), incorporating four new items. The IBIS-R assesses ideal female body stereotypes by asking participants for their level of agreement with 10 statements concerning what attractive women look like on a 5-point scale ranging from strongly agree to strongly disagree.

The second measure assessing internalization of body ideals is the SATAQ which has a number of different versions (Cusumano and Thompson 1997; Heinberg et al. 1995; Schaefer et al. 2015, 2017; Thompson et al. 2004). Although the SATAQ (Heinberg et al. 1995) was initially developed to assess factors of awareness (i.e., recognition or acknowledgement of 
societal appearance norms) and internalization (i.e., to assume these norms as an individual's own), the instrument has been expanded from the initial 14 items (six items for the awareness factor, and eight items for the internalization factor) up to 28 items in the most recent version (SATAQ-4R; Schaefer et al. 2017). These new items assess social pressure factors from different sources (i.e., family, peers, significant others, and media) and internalization of different body ideals (i.e., thin/low body fat, muscular body, and general attractiveness). However, despite internalization of body ideals being identified as a risk factor in the literature, these instruments have some specific limitations.

First, the instruments that assess the internalization construct have not used the same definition as the construct itself. For instance, although the IBIS-R title reflects a measure of internalization, the drafting of its items is more a recognition of a social ideal of appearance (e.g., "Slender women are more attractive," "Tall women are more attractive") and these items are therefore closer to those employed to assess the SATAQ awareness factor (e.g., "Attractiveness is very important if you want to get ahead in our culture") (Heinberg et al. 1995) and SATAQ-R (e.g., "In our culture, someone with a well-built body has a better chance of obtaining success") (Cusumano and Thompson 1997). On the other hand, the SATAQ internalization concept has varied across its different versions, from a general internalization or general attractiveness, to the differentiation of two specific types of internalization (i.e., thin/low body fat and muscular body). Nonetheless, researchers have traditionally used the internalization-general subscale as a measure of thin-ideal internalization (Cusumano and Thompson 1997; Heinberg et al. 1995; Thompson et al. 2004), which could lead to an overlap with some of the specific types of internalization both conceptually and empirically (Schaefer et al. 2015). Consequently, the desire for an attractive appearance measurement would involve a barely sustainable assumption that all respondents interpret items referring to media ideals in an abstract way, without referencing any of the specific socially sanctioned beauty ideals (i.e., thin/low body fat, muscular body), thus adding ambiguity to the internalization subscales' measurement.

Second, including the internalization construct measurement together with measurements for other constructs from sociocultural models makes it difficult to isolate the treatment of the internalization measure. Consequently, this may lead to an overlap with constructs assessed in other existing instruments. For example, when proposing the SATAQ-4, Schaefer et al. (2015) drafted the items for the muscular/athletic subscale assuming a wide definition for the internalization of appearance ideals. This encompassed not only cognitive processes, that is, the degree to which an individual "buys into" socially prescribed appearance ideals and expresses a desire to attain the appearance ideal (e.g., "I think a lot about looking muscular"), but also behavioral elements of the construct, namely, engaging in behaviors aimed at meeting those ideals (e.g., "I spend a lot of time doing things to look more athletic"). In an attempt to clean up the muscular/athletic subscale measurement, Schaefer et al. (2017) recently revised the SATAQ-4 and removed the behavioral items from the muscularity internalization subscale. This is because there are other instruments that assess such behaviors including the Drive for Thinness subscale, in the third edition of the Eating Disorder Inventory (EDI-3; Garner 2004), the Drive for Muscularity Scale (McCreary and Sasse 2000), and the Body Change Inventory (Ricciardelli and McCabe 2002).

Although the rewriting of items in the SATAQ-4 now provides a purer delimitation of the internalization construct, the instrument continues to encompass the assessment of other constructs defined in sociocultural models, such as appearance pressure subscales emanating from different sources (e.g., family, peers, media, and significant others) in the same instrument as the perceived pressure instrument, but not with other constructs that might form part of 
the sociocultural models (e.g., drive for body ideals, awareness). Furthermore, given that there are instruments that assess perceived pressure from different sources for having a thin body or muscular body, such as the Perceived Sociocultural Pressures Scale (PSPS; Stice et al. 1996; Tylka 2011), a unique instrument that assesses internalization of body ideals would help researchers to examine the relationship between this construct and other variables in sociocultural models.

Third, given that athletic expression might not only involve muscularity but also denote a set of other physical attributes arising in the absence of muscularity, Schaefer et al. (2017) replaced the word "athletic" with "muscular" when revising the SATAQ-4 for items used to assess the internalization of a muscular ideal. However, the "muscular" ideal may also be interpreted as brawny, or as excessive muscular development, moving away from the typical mesomorphic body pattern. Including both "athletic" and "muscular" expressions in items assessing internalization of a muscular/athletic ideal would allow a wider and more inclusive evaluation of muscularity internalization.

Fourth, the drafting of some items in the SATAQ-4R internalization subscales does not suggest a desire to attain a particular body type as a consequence of constant social reinforcement of prominent appearance ideals from the media or other social agents. For example, items such as "I think a lot about having very little body fat" may reflect the desire to attain a thin body from the pressure received from socially prescribed appearance ideals but could also involve a desire to lose weight from following medical advice. Therefore, the drafting of items assessing internalization should capture the level to which the individual desires to attain particular appearance ideals (e.g., thin or muscular/athletic body) are a consequence of "buying into" socially prescribed appearance ideals.

Fifth, from a pool of 40 items, the SATAQ-4R (Schaefer et al. 2017) retains negative items for some factors including a negative item for the muscular subscale ("I don't want my body to look muscular") in the version validated with female college students and adolescent girls. However, the inclusion of a few negative items in an otherwise positively stated questionnaire can lead to ambiguous results rather than controlling response sets (Roszkowski and Soven 2010). Moreover, the inclusion of negatively worded items has proven especially problematic with young sample groups and its subsequent translation into other languages has proved to be particularly troublesome (Wong et al. 2003). Consequently, it seems sensible to devise an internalization assessment that does not mix the wording of negative and positive items.

Finally, most of the instruments for assessing the internalization of specific types of body ideals were originally developed for exclusive use by females. Only the last two versions of the SATAQ have tested the instrument's structure in samples of both men and women. However, in both cases, it started from the assumption that the instrument's factorial structure will vary significantly between males and females as a result of gender differences in body image concerns. Consequently, measurement invariance of the assessed constructs between females and males was not tested. Schaefer et al. (2017) began with an initial pool of 40 items for the SATAQ-4R and underwent separate exploratory and confirmatory factor analyses employing a sample of female college students (study 1) and male college students (study 3). As a result of these analyses, the authors proposed a 31-item version of the SATAQ-4R for women and a 28item version for men, which impeded simultaneous use of the instrument on sample groups of men and women.

To date, it has been assumed that women are more sensitive to internalizing a thin body ideal while men are more sensitive to internalizing a muscular body ideal (Thompson et al. 2012). However, comparisons between males and females with the same instrument are 
difficult without having prior certainty that the instrument's items possess the same meaning, and that the underlying constructs (e.g., thin and muscular body internalization) are perceived similarly in both groups (Brown 2015). The equivalence/invariance measurement of an instrument assessing thin and muscular body internalization would allow comparison between males and females. This approach could affirm that the reported differences are indicative of true gender differences, thus avoiding inaccurate practical inferences.

Taking into account the aforementioned limitations, the purpose of the present study was to review the different versions of SATAQ to (a) isolate an assessment for the internalization of thin and muscular/athletic body ideals from other sociocultural risk factors for body image disturbance and eating disorders; (b) reduce the ambiguity of the assessment by not including reverse-worded items; (c) make the internalization process clearer to reflect "buying into" some of the socially sanctioned body ideals; (d) examine the factorial structure, the reliability, and the measurement invariance across a large community sample of 10- to 14-year-old females and males; and (e) examine criterion validity based on the relationship between internalization of sociocultural body ideals and social-physique anxiety (SPA).

Concerning criterion validity, it was hypothesized that, once gender and body mass index (BMI) effects were controlled for, internalization of the thin body ideal would have a greater predictive effect on SPA than internalization of the muscular/athletic body ideal. To date, it has not been possible to precisely test this relationship given that no internalization instrument has been developed that was invariant across gender. Indeed, the literature has shown that girls usually present greater SPA levels than boys (Sáenz-Álvarez et al. 2013), so the relationship between body ideal internalization and SPA might be affected in part by gender.

\section{Method}

\section{Item Generation}

Taking into account the items of the SATAQ-4R (and its previous versions), an initial pool of 10 items (five for thin/low body fat body internalization and five for muscular/athletic body internalization) was generated by the authors to assess the internalization construct and address the aforementioned limitations. All of the items were drafted positively, avoiding the mixing of negative stems with positive stems, which might have introduced artifacts and increased result ambiguity (Roszkowski and Soven 2010). To assess the internalization of a muscular/athletic ideal, items that only used the word "athletic" or "muscular" were not included to avoid less inclusive interpretations than those that might be understood for a mesomorphic body. Finally, the present authors avoided drafting items that did not capture the cognitive process of "buying into" socially prescribed appearance ideals implicit in the internalization construct (e.g., "I think a lot about having very little body fat").

\section{Participants}

The sample comprised 1130 students (48\% girls) aged between 10 and 14 years $(M=11.53$ years, $S D=0.71)$ with a mean self-reported body mass index $(\mathrm{BMI})$ of $19.01 \mathrm{~kg} / \mathrm{m}^{2}\left(S D_{B M I}=\right.$ 3.61) from different education centers in two Spanish provinces. In the temporal stability analysis, an independent sample of 52 students (50\% girls) aged between 12 and 15 years was used $(M=12.78$ years, $S D=0.83)$. 


\section{Measures}

Internalization of Sociocultural Body Ideals Scale The preliminary 10 items generated from the revised SATAQ-4R and from its previous versions were used to assess the internalization of the Thin Body Ideal (TBI) (i.e., the desire to achieve as thin a body as the socially accepted stereotype) and internalization of the Muscular/Athletic Body Ideal (MBI) (i.e., the desire to achieve a body as athletic and muscular as the socially accepted stereotype). Responses were provided on a Likert scale ranging from 1 (totally disagree) to 5 (totally agree).

SPA Scale The Spanish version (Sáenz-Álvarez et al. 2013) of the Social Physique Anxiety Scale (SPAS-7; Motl and Conroy 2000) comprises six items grouped into one factor (e.g., "It would make me uncomfortable to know others were evaluating my physique/figure"). Responses were provided on a Likert scale ranging from 1 (never) to 5 (always). One item 5 was reverse-coded, and higher scores correspond to greater levels of SPA. In the present study, a construct reliability value ( $H$; Hancock and Mueller 2001) of 0.86 was obtained.

\section{Procedure}

Prior to data collection, the ISBIS was pilot tested on a small group of nine students aged between 11 and 14 years. They were asked to identify any item whose meaning was not clear to them. None of the participants claimed not to understand any of the proposed items. Following this, the directors of the educational centers were contacted to request their collaboration in the study. The participants, for whom authorization from parents or legal guardians had previously been obtained, were informed about the anonymous and voluntary nature of their participation. The questionnaire was completed in school classrooms in the presence of one of the research team. The Ethics Committee of the first author's institution approved the study protocol.

\section{Data Analyses}

The total sample was split randomly into equal halves $(n=565)$. The first subsample $(48 \%$ girls; $M=11.56$ years, $S D=0.73$ ) was used to perform an exploratory factor analysis (EFA), while the second subsample ( $48 \%$ girls; $M=11.51$ years, $S D=0.69$ ) was employed in the subsequent confirmatory factor analysis (CFA). Factorial analyses were conducted using the weighted least squares adjusted by mean and variance (WLSMV) estimation method and the polychoric correlation matrix (Muthén and Muthén 1998-2015). This estimation method allows obtaining a standard error that is robust to non-normality with ordinal polytomous variables (Li 2015). Given that the data were collected in different educational centers, and to avoid the likely non-independence of the observations, the cluster option and the COMPLEX function of Mplus were used.

On the basis of the theoretically correlated nature of the instrument's constructs, and to facilitate the comparison of the factorial structure obtained in the CFA with that obtained in the EFA (Schmitt and Sass 2011), the latter analysis was conducted using an oblique Geomin rotation $(\varepsilon=0.5$ ) (Morin et al. 2013). In the absence of secondary factor loadings $>0.32$, primary factor loadings $>0.50$ were considered adequate (Costello and Osbourne 2005). Goodness-of-fit was judged on the following indicators: 
The ratio between $\chi^{2}$ and degrees of freedom $\left(\chi^{2} / d f\right)$, the comparative fit index (CFI), the Tucker-Lewis index (TLI), the root mean square error of approximation (RMSEA) with its $90 \%$ confidence interval (CI), and the weighted root mean square residual (WRMR). According to the criteria proposed for the CFA involving ordinal polytomous variables (Yu 2002), values $\geq 0.96$ for CFI and TLI; $\geq 0.95, \leq 0.05$ for RMSEA; and $\leq 0.95$ for WRMR were considered as indicative of an excellent data fit to the model. After verifying the factorial structure in the CFA, which was previously identified in the EFA, both subsamples were combined with the total sample being employed in the subsequent analyses.

To provide evidence that supports the invariant character of the ISBIS across gender, the procedure proposed by Muthén and Muthén (1998-2015) for ordered polytomous variables was followed. First, the equivalence of the factorial structure between groups was tested (i.e., the configural invariance). To fulfill this goal, the model was tested: (a) independently in girls (M0a) and boys (M0b) and (b) simultaneously (i.e., specifying a multigroup analysis) for girls and boys (M0). Then, a model nested to the latter (M1) was tested. Given that the response probability curve of the item is influenced by both its factorial loading and thresholds, the two parameters were simultaneously constrained in this model (M1) (Muthén and Muthén 1998-2015). Significant differences $(p<0.05)$ between the nested models in the DIFFTEST procedure of the Mplus (i.e., a modified form of testing nested models employing the WLMSV estimation method) were considered as indicative of non-invariance.

Subsequently, the present study obtained (a) the construct reliability for the two factors employing the $H$ coefficient (Hancock and Mueller 2001); (b) the average extracted variance (AVE); and (c) the temporal stability employing the intra-class coefficient, and its $95 \% \mathrm{CI}$ was obtained by specifying a bi-directional model with an absolute agreement definition. Values $\geq$ 0.70 for $H$ and CCI, and $\geq 0.50$ for AVE were considered adequate (Fleiss 2011; Hair et al. 2006). To obtain evidence supporting the instrument's discriminant validity, the heterotraitmonotrait (HTMT) ratio of correlations between factors was obtained with values $<0.85$ being considered as adequate (Henseler et al. 2015).

To provide evidence of criterion validity, a linear regression analysis was conducted in which gender and BMI (Step 1) and internalization factors (Step 2) were introduced as independent variables, and SPA as the dependent variable. In this analysis, the gender variable was codified as ' 0 ' (girls) and ' 1 ' (boys). A statistical significance level of 0.05 was employed. The factorial analyses were conducted in Mplus 7 whereas IBM-SPSS-22 was employed in the remaining analyses.

\section{Results}

\section{Exploratory Factor Analysis}

The results of the first EFA (Table 1) showed a secondary factor loading greater than 0.32 for item 6 (English translation: "I compare my body to those of people who are athletic and muscular"), in particular, in the boys' subsample. Once item 6 was removed, a second EFA showed a factor loading greater than 0.32 for item no. 9 (English translation: "I compare the size of my body with those of thin people that appear in magazines"), in the boys' subsample. Once item 9 was removed, the results of the third 
Table 1 Exploratory factor analysis of the ISBIS

\begin{tabular}{|c|c|c|c|c|c|c|c|c|c|c|c|c|}
\hline & \multicolumn{4}{|c|}{ EFA 1} & \multicolumn{4}{|c|}{ EFA 2} & \multicolumn{4}{|c|}{ EFA 3} \\
\hline & \multicolumn{2}{|l|}{ Girls } & \multicolumn{2}{|l|}{ Boys } & \multicolumn{2}{|l|}{ Girls } & \multicolumn{2}{|l|}{ Boys } & \multicolumn{2}{|l|}{ Girls } & \multicolumn{2}{|l|}{ Boys } \\
\hline & $\mathrm{F} 1$ & $\mathrm{~F} 2$ & $\mathrm{~F} 1$ & $\mathrm{~F} 2$ & $\mathrm{~F} 1$ & $\mathrm{~F} 2$ & $\mathrm{~F} 1$ & $\mathrm{~F} 2$ & $\mathrm{~F} 1$ & $\mathrm{~F} 2$ & $\mathrm{~F} 1$ & $\mathrm{~F} 2$ \\
\hline Item 1 & 0.63 & 0.28 & 0.58 & 0.23 & 0.60 & 0.31 & 0.57 & 0.26 & 0.62 & 0.29 & 0.59 & 0.25 \\
\hline Item 2 & 0.10 & 0.69 & 0.03 & 0.86 & 0.05 & 0.75 & 0.02 & 0.88 & 0.07 & 0.73 & 0.04 & 0.87 \\
\hline Item 3 & 0.81 & 0.16 & 0.93 & 0.02 & 0.80 & 0.18 & 0.93 & 0.01 & 0.83 & 0.14 & 0.92 & 0.03 \\
\hline Item 4 & 0.14 & 0.76 & 0.08 & 0.77 & 0.15 & 0.73 & 0.11 & 0.73 & 0.18 & 0.71 & 0.12 & 0.72 \\
\hline Item 5 & 0.73 & 0.14 & 0.62 & 0.26 & 0.71 & 0.16 & 0.61 & 0.30 & 0.73 & 0.14 & 0.56 & 0.31 \\
\hline Item 6 & 0.15 & 0.80 & 0.37 & 0.52 & & & & & & & & \\
\hline Item 7 & 0.82 & 0.17 & 0.84 & 0.11 & 0.80 & 0.20 & 0.85 & 0.11 & 0.80 & 0.19 & 0.85 & 0.12 \\
\hline Item 8 & 0.32 & 0.65 & 0.18 & 0.75 & 0.27 & 0.70 & 0.17 & 0.78 & 0.30 & 0.68 & 0.19 & 0.77 \\
\hline Item 9 & 0.77 & 0.16 & 0.65 & 0.30 & 0.76 & 0.17 & 0.63 & 0.33 & & & & \\
\hline Item 10 & 0.17 & 0.73 & 0.26 & 0.64 & 0.15 & 0.74 & 0.27 & 0.63 & 0.16 & 0.74 & 0.28 & 0.63 \\
\hline
\end{tabular}

$\mathrm{F} 1$ = internalization of the thin body ideal, F2 = internalization of the muscular/athletic body ideal. Factor loadings $>0.50$ (primary) and $>0.32$ (secondary) are italicized

EFA showed primary factorial loadings ranging from 0.56 to 0.92 , as well as no secondary factor loadings greater than 0.32 . The two-factor model comprising four items per factor showed the following fit index values: $\chi^{2}(13, n=269)=24.28, p=0.03 ; \chi^{2} / g l$ $=1.87 ; \mathrm{CFI}=0.994 ; \mathrm{TLI}=0.987 ; \mathrm{RMSEA}=0.057(90 \% \mathrm{CI}=0.018,0.091)$ for girls, and $\chi^{2}(13, n=296)=22.18, p=0.05 ; \chi^{2} / g l=1.71 ; \mathrm{CFI}=0.989 ;$ TLI $=0.976 ;$ RMSEA $=0.049(90 \% \mathrm{CI}=0.000,0.083)$ for boys.

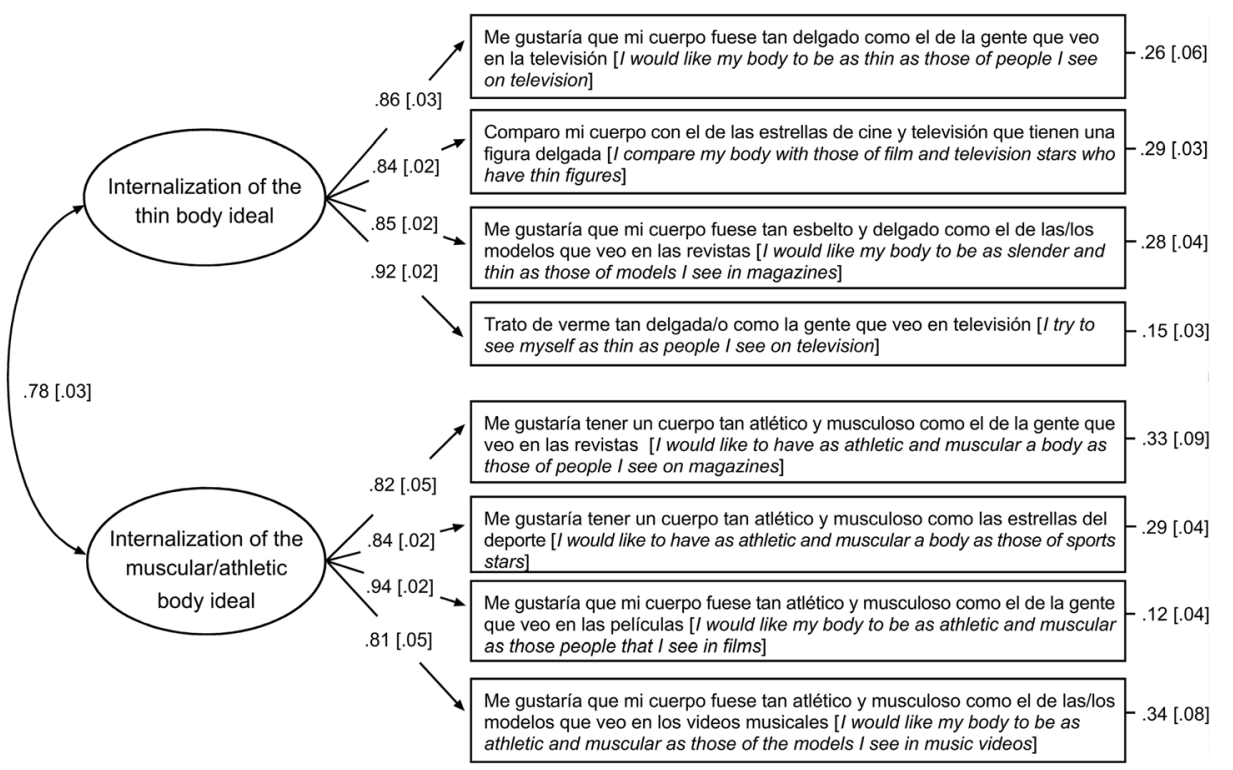

Fig. 1 Confirmatory factor analysis of the ISBIS. Ellipses represent latent factors and rectangles the items. Values inside brackets correspond to the standard errors. Values outside brackets at the right of the rectangles correspond to the error terms. All factor loadings and correlations shown were statistically significant $(p<0.001)$ 


\section{Confirmatory Factorial Analysis}

In the CFA conducted on the second subsample (Fig. 1), the two-factor model comprising four items per factor showed the following fit index values: $\chi^{2}(51, n=565)=31.88, p=0.03 ; \chi^{2} / g l$ $=0.63 ; \mathrm{CFI}=0.993 ; \mathrm{TLI}=0.990 ; \mathrm{RMSEA}=0.035(90 \% \mathrm{CI}=0.010,0.055) ; \mathrm{WRMR}=0.41$.

\section{Invariance Analyses}

The results of the invariance analyses showed adequate model fit index values for the models specified individually for girls and boys, as well as for the multigroup model, in which both subgroups were considered simultaneously. Additionally, no significant differences between M0 and M1 were obtained ( $p=0.17$ ) (Table 2).

\section{Reliability Analyses}

Values of $H=0.93$ and AVE $=0.75$ were obtained for the internalization of TBI, and $H=0.93$ and $\mathrm{AVE}=0.72$ for the internalization of MBI. The temporal stability analysis revealed ICC values of $0.90(95 \% \mathrm{CI}=0.67,0.96)$ for $\mathrm{TBI}$, and $0.98(95 \% \mathrm{CI}=0.94,0.99)$ for $\mathrm{MBI}$. The HTMT value for the correlation between factors was 0.78 .

\section{Criterion Validity Analysis}

The results in Table 3 show that, with the effects of gender and BMI controlled for, internalization of TBI $(M=1.94, S D=1.10$, asymmetry $=1.20$ and skewness $=0.43)$ predicted positively and in a statistically significant way SPA $(\beta=0.40, p<0.001)$. In the case of internalization of MBI $(M=2.15, S D=1.12$, asymmetry $=0.91$ and skewness $=0.01)$, this predictive effect was not statistically significant $(\beta=0.04, p=0.339)$. The proposed model explained $23 \%$ of the SPA variance.

\section{Discussion}

The present study describes the development and validation of the Internalization of Sociocultural Body Ideals Scale (ISBIS) in a sample of male and female preadolescents. Based on a

Table 2 Invariance analyses across gender

\begin{tabular}{|c|c|c|c|c|c|c|}
\hline Model & $\chi^{2}(d f)$ & $\begin{array}{l}\text { M0 vs M1 } \\
\text { DIFFTEST }(p)\end{array}$ & CFI & TLI & RMSEA (90\% CI) & WRMR \\
\hline M0a: only girls $(n=539)$ & $29.04(19)$ & & 0.998 & 0.996 & $0.031(0.000,0.053)$ & 0.49 \\
\hline M0b: only boys $(n=591)$ & $40.44(19)$ & & 0.982 & 0.974 & $0.044(0.030,0.062)$ & 0.47 \\
\hline $\begin{array}{l}\text { M0: base model } \\
\quad \text { (girls and boys) }\end{array}$ & $62.12(38)$ & & 0.995 & 0.993 & $0.034(0.017,0.048)$ & 0.67 \\
\hline $\begin{array}{l}\text { M1: M0 + invariant factor } \\
\text { loadings and thresholds }\end{array}$ & $85.77(66)$ & 0.17 & 0.996 & 0.997 & $0.023(0.000,0.036)$ & 0.87 \\
\hline
\end{tabular}

CFI comparative fit index, TLI Tucker-Lewis index, RMSEA root mean square error of approximation, $C I$ confidence interval, WRMR weighted root mean square residual 
Table 3 Linear regression analysis predicting SPA from gender and internalization of the body ideals

\begin{tabular}{llllll}
\hline & $B$ & $\mathrm{SE} B$ & $\beta$ & $p$ & $R^{2}$ \\
\hline Step 1 & & & & & 0.04 \\
$\quad$ Sex & -0.11 & 0.04 & -0.07 & 0.013 & \\
$\quad$ BMI & 0.04 & 0.01 & 0.20 & $<0.001$ & 0.23 \\
Step 2 & & & & & \\
$\quad$ Sex & -0.10 & 0.04 & -0.07 & 0.011 \\
$\quad$ BMI & 0.03 & 0.01 & 0.13 & $<0.001$ \\
Internalization of the thin-body ideal & 0.26 & 0.02 & 0.40 & $<0.001$ \\
Internalization of the muscular/athletic body ideal & 0.02 & 0.02 & 0.04 & 0.339 \\
\hline
\end{tabular}

$B$, unstandardized regression coefficients; $S E$, standard error; $\beta$, standardized regression coefficients; $R^{2}$, explained variance. Sex was codified as girls $=0$ and boys $=1$

review of items in the previous versions of the Sociocultural Attitudes Towards Appearance Questionnaire (SATAQ), the ISBIS considers the internalization measure in isolation as one of the sociocultural risk factors for body disturbance and eating disorders. This provided a more precise assessment of this construct for a number of reasons. Firstly, by only considering the internalization measure, the relationship of this construct can be analyzed alongside other sociocultural factors that might be assessed using other existing instruments. Secondly, it focuses on the two specific body ideals examined in the literature (Thompson et al. 2012), avoiding overlaps with more general definitions of the construct. Thirdly, based on the accepted definition of the construct, it makes the internalization of body ideal process clear as a reflection of "buying into" the socially sanctioned appearance ideals. Fourthly, eliminating reverse-worded items likely reduces carelessness or misunderstanding and, therefore, ambiguity in the response to the measure. Finally, it offers a single instrument, with the items from the different constructs retaining the same meaning for males and females, and therefore allowing a comparison of the internalization of sociocultural body ideals between both groups.

The development of the ISBIS began with an initial pool of 10 items that assessed the cognitive elements for the internalization of TBI and MBI and brought together a process that reflected the "buying into" socially sanctioned appearance ideals. Although all of the items demonstrated primary factorial saturation $>0.50$ in their factor, a restrictive criterion was applied in the explanatory factorial analysis (Costello and Osbourne 2005 ), and two items were eliminated for showing secondary saturation $>0.32$. As a result of successive EFA, the structure of the eight-item model and two internalization factors was suggested and later confirmed through CFA that provided excellent fit indexes. The correlation between the TBI and MBI internalization factors was moderately high, suggesting that the two constructs are not completely exclusive, and supporting the idea that they might be present both for girls and boys, in such a way that an individual might simultaneously desire some parts of their body to be thinner (e.g., stomach and waist) yet stronger in other parts (e.g., arms and legs) (Bozsik et al. 2018; Thompson et al. 2012, 2004). Moreover, the results of the internal consistency and temporal stability analyses were adequate, suggesting that the ISBIS is a valid and reliable instrument to assess internalization for TBI and MBI in preadolescents.

On the other hand, the multigroup analysis provided evidence suggesting that the factor structure of the ISBIS was invariant across gender. These results support the future use of ISBIS for comparing TBI and MBI between males and females, which provides a distinct advantage with respect to the existing versions of the SATAQ. Validation of the different 
versions of SATAQ were carried out exclusively on samples of women (Thompson et al. 2004) or in studies on women and men, but belonging to independent sample groups (Schaefer et al. 2015; Schaefer et al. 2017). This has impeded the examination of a factorial structure that was invariant for both groups. The multigroup analysis carried out in the present study provides evidence that the eight items of the ISBIS assessing thin-ideal and muscular/athletic-ideal constructs are interpreted in a similar way by both males and females. The ISBIS will allow future research to challenge many of the assumptions that have been established for the appearance ideals that boys and girls maintain. For example, past research has reported that thin-ideal internalization maintains an association with or predicts SPA (Dittmar and Howard 2004). However, given that the literature has also found greater SPA scores in girls than in boys (Sáenz-Álvarez et al. 2013), it was difficult to ensure that the association between TBI internalization and SPA was not because of a gender influence. In this regard, the results obtained using an instrument like the ISBIS, which shows invariance across gender, demonstrate that, once the effect of sex and BMI are controlled for, the internalization of TBI had a predictive effect on SPA. These results clarify the relationship between the variables, as they affirm that, apart from the effect that gender may have on SPA, the internalization of TBI shows a greater predictive effect on SPA than does the internalization of MBI. Having an instrument that is invariant across gender will allow future research to make comparisons between the appearance ideals of males and females and the relationship of these variables with other variables.

Although the results of this study lend psychometric support to ISBIS, there are some limitations that should be noted when interpreting the findings. Firstly, the sample group used comprised Spanish preadolescent students. Therefore, future studies need to evaluate the scale with other populations (e.g., adults, adolescents, clinical populations) in other countries and cultures. Furthermore, the sample was not probabilistic, so the results cannot be generalized, and further studies are necessary with students of different ages and evolving characteristics. Secondly, the present study focused upon the analysis of the psychometric properties of the ISBIS and demonstrated evidence of criterion validity by analyzing the relationship of the subscales with SPA. Future studies need to consider other associations with variables that are psychological (e.g., body dissatisfaction), biological (e.g., puberty), and sociocultural (e.g., social pressure) in nature to provide more evidence of criteria validity.

In spite of the aforementioned limitations, the ISBIS demonstrated that it is a valid and reliable instrument for assessing the internalization of TBI and MBI in both male and female preadolescents. The study contributes to the scientific field by developing an instrument (a) with strong internal structure, (b) which is useful and easy to use, and (c) that assesses one of the key sociocultural risk factors for psychological disorders (e.g., body dissatisfaction) or eating disorders (Dye 2016; Thompson and Stice 2001). The development of a scale that assesses the internalization construct for an ideal body both in boys and in girls is of great interest, given that this construct has been indicated as a risk factor in eating disorders and body dissatisfaction. Furthermore, being able to use the instrument with preadolescents helps in the early detection of the internalization of the social ideals of beauty, which will also help in preventing eating pathologies and body image disturbance (Dittmar and Howard 2004).

Funding Information "This research was funded by Ministerio de Economía y Competitividad and FEDER funds: grant number DEP2014-57228-R." Also, this work was supported by the first author's grant from the Spanish Ministry of Education, Culture and Sport through the Sate's Mobility Subprogram of the National Plan for Research, Development and Innovation (Ref. PRX18/00351). 


\section{Compliance with Ethical Standards}

Ethical Approval All procedures performed in this study involving human participants were in accordance with the ethical standards of University's Research Ethics Board and with the 1975 Helsinki Declaration.

Informed Consent Informed consent was obtained from all participants.

Conflict of Interest The authors declare that they have no conflict of interest.

Open Access This article is distributed under the terms of the Creative Commons Attribution 4.0 International License (http://creativecommons.org/licenses/by/4.0/), which permits unrestricted use, distribution, and reproduction in any medium, provided you give appropriate credit to the original author(s) and the source, provide a link to the Creative Commons license, and indicate if changes were made.

\section{References}

Bassett-Gunter, R., McEwan, D., \& Kamarhie, A. (2017). Physical activity and body image among men and boys: A meta-analysis. Body Image, 22, 114-128. https://doi.org/10.1016/j.bodyim.2017.06.007.

Bozsik, F., Whisenhunt, B. L., Hudson, D. L., Bennett, B., \& Lundgren, J. D. (2018). Thin is in? Think again: The rising importance of muscularity in the thin ideal female body. Sex Roles, (online), 1-7. https://doi. org/10.1007/s11199-017-0886-0.

Brown, T. A. (2015). Confirmatory factor analysis for applied research (2nd ed.). New York: Guilford Publications.

Costello, A., \& Osbourne, J. W. (2005). Best practices in exploratory factor analysis: four recommendations for getting the most from your analysis. Practical Assessment, Research \& Evaluation, 10(7), 1-9.

Cusumano, D. L., \& Thompson, J. K. (1997). Body image and body shape ideals in magazines: Exposure, awareness, and internalization. Sex Roles, 37(9-10), 701-721. https://doi.org/10.1007/BF02936336.

Dakanalis, A., Timko, A. C., Clerici, M., Riva, G., \& Carra, G. (2017). Objectified body consciousness (OBC) in eating psychopathology: Construct validity, reliability, and measurement invariance of the 24-item OBC scale in clinical and nonclinical adolescent samples. Assessment, 24(2), 252-274. https://doi.org/10.1177 $/ 1073191115602553$.

Dittmar, H., \& Howard, S. (2004). Thin-ideal internalization and social comparison tendency as moderators of media models' impact on women's body-focused anxiety. Journal of Social and Clinical Psychology, 23(6), 768-791. https://doi.org/10.1521/jscp.23.6.768.54799.

Dye, H. (2016). Does internalizing society and media messages cause body dissatisfaction, in turn causing disordered eating? Journal of Evidence-Informed Social Work, 13(2), 217-227. https://doi.org/10.1080 /23761407.2015.1024572.

Edwards, C., Tod, D., Molnar, G., \& Markland, D. (2016). Perceived social pressures and the internalization of the mesomorphic ideal: The role of drive for muscularity and autonomy in physically active men. Body Image, 16, 63-69. https://doi.org/10.1016/j.bodyim.2015.11.003.

Fleiss, J. L. (2011). Design and analysis of clinical experiments. New York: John Wiley \& Sons. https://doi. org/10.1002/9781118032923.

Garner, D. M. (2004). Eating disorder inventory-3 professional manual. Odessa: Psychological Assessment Resources, Inc..

Hair, J. F., Black, B., Babin, B. J., Anderson, R. E., \& Tatham, R. L. (2006). Multivariate data analysis (7th ed.). Upper Saddle River: Pearson Prentice Hall.

Hancock, G. R., \& Mueller, R. O. (2001). Rethinking construct reliability within latent variable systems. In R. Cudeck, S. du Toit, \& D. Sörbom (Eds.), Structural equation modeling: present and future (pp. 195-216). Lincolnwood: Scientific Software International.

Heinberg, L. J., Thompson, J. K., \& Stormer, S. (1995). Development and validation of the Sociocultural Attitudes Towards Appearance Questionnaire. International Journal of Eating Disorders, 17(1), 81-89. https://doi.org/10.1002/1098-108X(199501)17:1<81::AID-EAT2260170111>3.0.CO;2-Y. 
Henseler, J., Ringle, C. M., \& Sarstedt, M. (2015). A new criterion for assessing discriminant validity in variancebased structural equation modeling. Journal of the Academy of Marketing Science, 43(1), 115-135. https://doi.org/10.1007/s11747-014-0403-8.

Karazsia, B. T., \& Crowther, J. H. (2008). Psychological and behavioral correlates of the SATAQ-3 with males. Body Image, 5(1), 109-115. https://doi.org/10.1016/j.bodyim.2007.08.004.

Li, C.-H. (2015). Confirmatory factor analysis with ordinal data: Comparing robust maximum likelihood and diagonally weighted least squares. Behavior Research Methods, 48, 936-949. https://doi.org/10.3758 /s13428-015-0619-7.

McCabe, M. P., Busija, L., Fuller-Tyszkiewicz, M., Ricciardelli, L. A., Mellor, D., \& Mussap, A. (2015). Sociocultural influences on strategies to lose weight, gain weight, and increase muscles among ten cultural groups. Body Image, 12(1), 108-114. https://doi.org/10.1016/j.bodyim.2014.10.008.

McCreary, D. R., \& Sasse, D. K. (2000). An exploration of the drive for muscularity in adolescent boys and girls. Journal of American College Health, 48(6), 297-304.

Morin, A. J. S., Marsh, H. W., \& Nagengast, B. (2013). Exploratory structural equation modeling. In G. R. Hancock \& R. O. Mueller (Eds.), Structural equation modeling: a second course (2nd ed., pp. 395-436). Charlotte: Information Age Publishing.

Motl, R. W., \& Conroy, D. E. (2000). Validity and factorial invariance of the Social Physique Anxiety Scale. Medicine and Science in Sports and Exercise, 32(5), 1007-1017. https://doi.org/10.1097/00005768200005000-00020.

Muthén, L. K., \& Muthén, B. O. (1998-2015). Mplus version 7: user's guide (7th ed.). Los Angeles: Muthén \& Muthén.

Ricciardelli, L. A., \& McCabe, M. P. (2002). Psychometric evaluation of the body change inventory: An assessment instrument for adolescent boys and girls. Eating Behaviors, 31(1), 45-59.

Ricciardelli, L. A., \& McCabe, M. P. (2004). A biopsychosocial model of disordered eating and the pursuit of muscularity in adolescent boys. Psychological Bulletin, 130(2), 179-205. https://doi.org/10.1037/00332909.130.2.179.

Roszkowski, M. J., \& Soven, M. (2010). Shifting gears: Consequences of including two negatively worded items in the middle of a positively worded questionnaire. Assessment \& Evaluation in Higher Education, 35(1), 117-134. https://doi.org/10.1080/02602930802618344.

Sáenz-Álvarez, P., Sicilia, Á., González-Cutre, D., \& Ferriz, R. (2013). Psychometric properties of the Social Physique Anxiety (SPAS-7) in Spanish adolescents. Spanish Journal of Psychology, 16(86), 1-9. https://doi. org/10.1017/sjp.2013.86.

Schaefer, L. M., Burke, N. L., Thompson, J. K., Dedrick, R. F., Heinberg, L. J., Dedrik, R. F., ... Swami, V. (2015). Development and validation of the Sociocultural Attitudes Towards Appearance Questionnaire-4 (SATAQ-4). International Journal of Eating Disorders, 27(1), 54-67. https://doi.org/10.1037/a0037917.

Schaefer, L. M., Harriger, J. A., Heinberg, L. J., Soderberg, T., \& Thompson, J. K. (2017). Development and validation of the Sociocultural Attitudes Towards Appearance Questionnaire-4-Revised (SATAQ-4R). International Journal of Eating Disorders, 50(2), 104-117. https://doi.org/10.1002/eat.22590.

Schmitt, T. A., \& Sass, D. A. (2011). Rotation criteria and hypothesis testing for exploratory factor analysis: implications for factor pattern loadings and interfactor correlations. Educational and Psychological Measurement, 71(1), 95-113. https://doi.org/10.1177/0013164410387348.

Stice, E. (2001). A prospective test of the dual-pathway model of bulimic pathology: mediating effects of dieting and negative affect. Journal of Abnormal Psychology, 110(1), 124-135. https://doi.org/10.1037/0021-843 X.110.1.124.

Stice, E., Schupak-Neuberg, E., Shaw, H. E., \& Stein, R. I. (1994). Relation of media exposure to eating disorder symptomology: An examination of mediating mechanisms. Journal of Abnormal Psychology, 103(4), 836840. https://doi.org/10.1037/0021-843X.103.4.836.

Stice, E., Ziemba, C., Margolis, J., \& Flick, P. (1996). The dual pathway model differentiates bulimics, subclinical bulimics, and controls: testing the continuity hypothesis. Behavior Therapy, 27(4), 531-549. https://doi.org/10.1016/S0005-7894(96)80042-6.

Stice, E., Shaw, H., \& Nemeroff, C. (1998). Dual pathway model of bulimia nervosa: Longitudinal support for dietary restraint and affect-regulation mechanisms. Journal of Social and Clinical Psychology, 17(2), 129-149.

Stice, E., Marti, C. N., \& Durant, S. (2011). Risk factors for onset of eating disorders: Evidence of multiple risk pathways from an 8-year prospective study. Behaviour Research and Therapy, 49(10), 622-627. https://doi. org/10.1016/j.brat.2011.06.009.

Thompson, J. K., \& Stice, E. (2001). Thin-ideal internalisation: Mounting evidence for a new risk factor for body image disturbance and eating pathology. Current Directions in Psychological Science, 10(5), 181-183. https://doi.org/10.1111/1467-8721.00144. 
Thompson, J. K., Van Den Berg, P., Roehrig, M., Guarda, A. S., \& Heinberg, L. J. (2004). The Sociocultural Attitudes Towards Appearance Scale-3 (SATAQ-3): Development and validation. International Journal of Eating Disorders, 35(3), 293-304. https://doi.org/10.1002/eat.10257.

Thompson, J. K., Schaefer, L. M., \& Menzel, J. (2012). Internalization of the thin-ideal and muscular-ideal. In T. F. Cash (Ed.), Encyclopedia of body image and human appearance (pp. 499-504). San Diego: Elsevier. https://doi.org/10.1016/B978-0-12-384925-0.00079-1.

Tylka, T. L. (2011). Refinement of the tripartite influence model for men: Dual body image pathways to body change behaviors. Body Image, 8(3), 199-207. https://doi.org/10.1016/j.bodyim.2011.04.008.

Wong, N., Rindfleisch, A., \& Burroughs, J. E. (2003). Do reverse worded items confound measures in crosscultural consumer research? The case of the material values scale. Journal of Consumer Research, 30, 7291. https://doi.org/10.1086/374697.

Yu, C. Y. (2002). Evaluating cutoff criteria of model fit indices for latent variable models with binary and continuous outcomes. Doctoral thesis, University of California, Los Angeles.

Publisher's Note Springer Nature remains neutral with regard to jurisdictional claims in published maps and institutional affiliations.

\title{
Affiliations
}

\section{Alvaro Sicilia $^{1,2} \cdot$ Antonio Granero-Gallegos ${ }^{1,2}$ - Manuel Alcaraz-lbáñez ${ }^{1,2}$. Mark D. Griffiths ${ }^{3}$ • Isabel Sánchez-Gallardo ${ }^{4}$ • Jesús Medina-Casaubón ${ }^{4}$}

\author{
Alvaro Sicilia
}

asicilia@ual.es

Antonio Granero-Gallegos

agranero@ual.es

Isabel Sánchez-Gallardo

Isabelsanchez@correo.ugr.es

Jesús Medina-Casaubón

jmedinac@ugr.es

1 Departament of Education, University of Almeria, Ctra. de Sacramento s/n - La Cañada de San Urbano, 04120 Almeria, Spain

2 Health Research Centre, University of Almería, Almeria, Spain

3 International Gaming Research Unit, Psychology Division, Nottingham Trent University, 50 Shakespeare Street, Nottingham NG1 4FQ, UK

4 Departament of Physical Education and Sports, University of Granada, Camino de Alfacar, 21-18071 Granada, Spain 